Y. C. Minh and E. F. van Dishoeck, eds.

\title{
Molecular Data Needs in Astrochemistry
}

T. J. Millar

Physics Department, UMIST, PO Box 88, Manchester M60 1QD, UK

C. M. Walmsley

Osservatorio Astrofisico di Arcetri, Largo E. Fermi 5, I-50125 Firenze, Italy

C. Rebrion-Rowe

PALMS, Equipe d'astrochemie experimentale, Université de Rennes 1, 35042 Rennes Cedex, France

L. d'Hendecourt

Institut d'Astrophysique Spatiale - CNRS 'Astrochimie Experimentale,' Campus d'Orsay, Bat. 121, 91405 Orsay Cedex, France

S. Saito

Institute for Molecular Science, Myodaiji, Okazaki 444, Japan

F. Rostas

Observatoire de Paris-Meudon, DAMAp et UMR 8588 du CNRS, 92195 Meudon Cedex, France

Abstract. The development of astrochemistry is inextricably linked to the generation of fundamental data. In this report, we discuss data needs in terms of astrochemical models, gas-phase kinetics, molecular excitation, optical and UV spectroscopy, solid-state chemistry, and millimeter and submillimeter wave spectroscopy.

\section{Introduction}

The study of molecular astrophysics allows one to probe conditions in the universe from its origin, through studies of deuterated molecules, which can be used to infer a lower limit on the primordial $\mathrm{D} / \mathrm{H}$ ratio, via the study of high-redshift galaxies, theoretically through the effects of $\mathrm{H}_{2}$ formation on the formation of protogalaxies (Hutchins 1976; Shapiro \& Kang 1987; Galli \& Palla 1998) and observationally through studies of CO emission (Guilloteau et al. 1997, 1999), to star formation in molecular clouds, the origin of our Solar System and planetary systems around other stars. 
To date, close to 120 molecules have been detected in interstellar and circumstellar clouds and they give rise to several thousand observed transitions. The interpretation of emission and absorption lines in terms of the underlying physics involves a number of disciplines. These include determining accurate transition frequencies and line strengths, in order to identify lines and to search for particular species; understanding collisional and radiative processes within molecules, traditionally a theoretical study but with increasing emphasis on experimental work; radiative transfer models, which couple the microscopic processes of absorption and emission to the macroscopic properties of the medium; and determining the reaction processes and rate coefficients needed to model the global chemical evolution of the medium. Thus, our understanding develops most rapidly by ensuring close collaboration between astronomers, spectroscopists, chemical physicists, and modellers, and involves both experimental and theoretical approaches.

In this panel discussion held at this Symposium, the aim was to concentrate on the important area of data needs over the time-scale to the next IAU Symposium on astrochemistry. The panel members were chosen to cover the areas of astrochemical models, gas-phase kinetics, molecular excitation, optical and UV spectroscopy, infrared solid-state spectroscopy, and millimeter and submillimeter wave spectroscopy. An electronic post-box was set up before the Symposium, and an actual post-box was used at the Symposium, to allow participants to raise particular questions or to identify particular data needs. Each panel member was asked to include, where possible, these issues in their brief presentations.

The WWW is rapidly growing into a powerful tool to access and search molecular data bases and to distribute astrochemical programs. References to relevant WWW addresses are made throughout this paper, and can also be found through the WWW page of the IAU Working Group on Astrochemistry <http://www.strw.leidenuniv.nl/ iau34/>.

\section{Collisional Rates (C. M. Walmsley)}

Theoretical and laboratory studies of molecular collision rates play a vital role in the interpretation of the vast flood of data currently emerging from both large ground based telescopes and satellite experiments such as ISO and SWAS. A useful overview of many of the current problems as well as a summary of the work done on many systems is given in the book by Flower (1990). Much work of course has gone on since then: it is interesting to note for example the flurry of publications concerning collisional excitation of molecular hydrogen (e.g. Flower 1998; Balakrishnan et al. 1999) as well as recent studies of HD (e.g. Roueff \& Flower 1999). The fact that in some cases there is not complete agreement between different authors suggests that there is still work to be done and indicates that independent studies of the same system are often useful. Another general comment is that much of the work to date on collisional rates for interstellar molecules has concerned collisions with para- $\mathrm{H}_{2}$ or helium. However, as results from ISO are making clear, ortho- $\mathrm{H}_{2}$ is often of comparable abundance to the para-form and neglecting this fact may cause large errors. An example is the difference in propensity rules for collisions with $\mathrm{NH}_{3}$ (Flower, Offer, \& 
Schilke 1990). Thus, computations of the collisional rates with the ortho form of $\mathrm{H}_{2}$ are clearly needed.

That apart, it is useful to note the increased importance in current research (as compared to a decade ago) of the study of hot or shocked regions surrounding areas of current star formation. This is partly the consequence of space missions such as ISO and SWAS which have presented us with the perhaps poisoned gift of a plethora of observations of different water transitions (e.g. van Dishoeck $\&$ van der Tak and Melnick, this volume). Interpreting these will require input from various sources but certainly one of these has to be reliable collisional rates. Another aspect of this problem stems from the fact that we are discovering spectral lines from an increasing number of vibrationally excited states (e.g. Wyrowski, Schilke, \& Walmsley 1999) and interpreting these will requirc understanding both possible radiative pumps and collisional effects.

Also, it is worth noting that there are a number of species which clearly need a detailed study. One of these is methanol (see Norris, this volume) for which a detailed study is badly needed both because of the importance of the maser emission and because potentially it is a very useful density probe (e.g. Johnston et al. 1992). Methanol is interesting moreover because it is a case where relevant laboratory data exist (Lees \& Hague 1974) allowing a check on the theoretical rates. Finally, the increasing amount of data for relatively complex species (e.g. methyl formate, ethyl cyanide) will eventually require a more sophisticated interpretation than simply plotting a 'Boltzmann diagram'. The implication of this is that one needs collisional rates.

\section{Gas-Phase Kinetics (C. Rebrion-Rowe)}

The discussion is restricted to temperatures below $300 \mathrm{~K}$.

\subsection{Ion-molecule reactions}

Ion-molecule reactions have been studied experimentally and theoretically for several decades, and form the best known domain of gas-phase kinetics. A large variety of experimental techniques is available (SIFT, drift tube, FALP, ion traps, ICR, CRESU, ...) and the temperature range goes down to a few $\mathrm{K}$ (Smith 1994). Very often these methods not only provide reaction rates, but also the nature of the products and in the best cases, branching ratios. The very low pressure in ion traps allows one to measure, for example, the radiative association reactions involving $\mathrm{H}_{2}$. From a theoretical point of view, capture theories work for fast reactions and the net increase of the reaction rate when the temperature decreases, being due to the dipole moment of the neutral, is very well represented by an analytical formula derived using the SACM method (Troe 1996). However, capture theories cannot predict the nature of the products, and experimental results are lacking for complex molecules (PAHs, and generally all molecules exceeding 20 atoms). Also, slow reactions do not seem to obey a general behaviour, although the reaction rate usually increases when the temperature decreases. A particular category concerns the slow reactions with $\mathrm{H}_{2}$, whose rates are erratic (can increase or decrease with $T)$. 


\subsection{Neutral-neutral reactions}

These reactions were thought to be slow because of an hypothetical activation barrier. Their experimental study at low temperature began in the 90's in supersonic jet experiments (Smith, Rowe, \& Sims 1996; Rowe et al., this volume). Presently, less than 30 reactions have been investigated, and these reactions always involve one stable species. The important point is that these reactions have demonstrated that they can be fast at low temperature, and therefore they should be included in the models when of astrochemical interest. If occasionally the products have been determined, branching ratios are mainly unknown. No work has been done on reactions between two unstable molecules $(\mathrm{CH}+\mathrm{H}$, for instance). The interaction between two neutrals is weaker than the ion-induced dipole interaction, and therefore more difficult to model. Up to now, no rule of thumb or analytical formula is available which can be used to guess the reaction rate of a given system, and the nature of the products cannot be predicted.

\subsection{Dissociative recombination}

Despite there being no thermal measurements of dissociative recombination rates below $80 \mathrm{~K}$, experimental data exist at low energies consisting of cross sections measured using 'heavy' ion storage rings. This relatively new tool (less than 10 years old) can also provide branching ratios, but is technically limited to light ions (mass less than $50 \mathrm{amu}$ ). The recombination of heavier ions has been investigated with the FALP-MS technique at room temperature, and the available data clearly show that there is no clear link between the character of the ion and the reaction rate (Mitchell \& Rebrion-Rowe 1997). Moreover, no logic seems to govern the nature of the dissociative recombination products and branching ratios (Andersen et al., this volume). All open channels can be present. The modelling of the dissociative recombination mechanism is very difficult, and many processes have been imagined to describe this mechanism. Theoretical approaches are generally limited to triatomic ions, and their predictive capabilities are rather limited.

\subsection{Electron attachment}

Over the last couple of years, people have begun to think about the possibility of negative ions being the carriers of some DIBs. For the moment, the method of formation of such ions remains unknown, though electron attachment is a likely possibility. Much experimental work has been performed on this topic (Dunning 1995), over a wide electron energy range, but not with molecules relevant for interstellar chemistry. One important point is that the reaction rate depends more on the vibrational state of the molecule than on the kinetic energy of the electron. It has to be said that many of the experimental results concern termolecular attachment (i.e., attachment in which the negative ion can be stabilised by collisions), and today no experiment is designed to perform radiative attachment measurements.

\subsection{Miscellaneous}

Databases do not provide information when the molecules become too large, especially at low temperatures. Performing gas-phase experiments with highly 
condensible species is a technical challenge, and the increase of the number of isomers complicates the interpretation of the results. This lack of data explains why in soot formation models, a lot of reaction rates involved in the formation of larger molecules (PAHs) are extrapolations (without scientific basis) of rates available for similar reactions of benzene. Everybody agrees that we must consider the grains as very important actors in the chemistry of dense clouds, but who knows how they are formed? Presumably not on grains! Before reaching the size of a grain, a molecular structure could grow from monomer to dimer, then trimer, etc. Measuring reaction rates involving small clusters (neutral or ionised) could be interesting, and may induce another type of chemistry. Last but not least, no experimental results exist on one of the simplest class of reactions: the spin-orbit relaxation (of $\mathrm{C}, \mathrm{C}^{+}, \ldots$ ) induced by collisions (with hydrogen, helium, ....).

\section{Interstellar Grains and Solid State Chemistry (L. d'Hendecourt)}

One of the highlights of the recent flux of observational data related to the chemistry of the interstellar medium comes from ISO spectroscopic data provided by the SWS instrument. Recall that the mid-infrared region of the spectrum is the unique means to reasonably assess the chemical composition of the solid state interstellar material. Indeed, even tiny grains are opaque to ultraviolet light and scattering effects dominate in the visible, masking the realm of the grain chemical composition. The availability of ISO spectral data over a continuous and extended wavelength range $(2.5-45 \mu \mathrm{m})$ allows one to seriously confront the observations with the numerous results obtained from careful laboratory simulations, particularly those on 'dirty molecular ices'. The detection by ISO of 'unobservable' molecular species, e.g. $\mathrm{CO}_{2}, \mathrm{CH}_{4}$ (de Graauw et al. 1996), largely predicted from laboratory simulations, shows clearly that elaborate gas phase models, even those including some grain chemical reaction schemes, must follow more closely the somehow empirical results derived from the laboratory simulations.

We are now in a position to build more accurate models of the chemical evolution of the interstellar gas in various environments but we must bear in mind, that, apart from $\mathrm{H}_{2}$ and $\mathrm{CO}$, most of the molecular material present in space resides in the solid state form (Dartois et al. 1998). Chemical evolution of this cold material upon photo- and thermo-processing, providing an out of equilibrium chemistry even in the solid phase, will be the challenge for the next decade.

Build-up of more complex organic species has been discussed in the past following ultraviolet or energetic particle irradiation of laboratory ices. However the efficiency and occurrence of this process in the reality of the interstellar medium is still far from quantitatively assessed. This is naturally an important issue since it appears that interstellar material is injected in protoplanetary environments, as some has indeed survived in comets or even in meteorites. Thus, it seems logical to assume that the complex chemistry that may have taken place on interstellar grains, may indeed have provided important building blocks for the onset of prebiotic materials at the surface of the Earth (or on some other possible telluric planets on other solar systems). This scenario is by no means 
fully understood or proven and the connections between interstellar chemistry and protoplanetary disk chemistry will be a primary goal for observations using FIRST and ALMA but also, on a much more intimate manner, for ROSETTA, which will measure numerous physical and chemical characteristics of a comet nucleus around 2012.

Another important result obtained by ISO-SWS, largely unexpected, is the observation that an important fraction of interstellar dust, particularly around evolved stars, is made of crystalline silicate materials. Thus, for the first time it seems possible to undertake seriously the mineralogy of interstellar dust (Waters et al. 1996). The precise ratio of crystalline to amorphous material is obviously related to some physical parameters (temperature and time of evolution), largely accessible from laboratory simulations. The beautiful similarity between some evolved star spectra with comet Hale-Bopp's spectrum strongly suggests some common evolution. Again, central to this problem will be the relationship of the interstellar chemical evolution and the formation of planetary disks and planets.

Finally, one must not forget surprising new results that may alter profoundly our view on interstellar dust formation and evolution. Although carbon is found in many different forms (from isolated PAHs to organic residues on silicate grains), the dominant form of carbon (most probably of interstellar origin), found in primitive meteorites is in the form of nanodiamonds of very small sizes (1.4 $\mathrm{nm}$ average in Orgueil). The recent interpretation of two infrared emission lines as coming from diamond emission (Guillois, Ledoux, \& Reynaud 1999) clearly shows that our knowledge of carbon grain nucleation in the atmospheres of cool stars must be revisited to account for this huge fraction of nanodiamonds in meteoritic materials. In particular, nanoparticle properties, a real challenge for the physicists in our laboratories, should be carefully considered by the astrophysicist, showing once again the real multidisciplinary approach which confronts our field. Here again, the connection between the interstellar matter and our Solar System (or other planetary systems) is at the center of these studies.

\section{Laboratory Millimeter and Submillimeter-Wave Spectroscopy ( $S$. Saito)}

Almost all of the 120 or so molecular species have been identified in space by radioastronomy in close collaboration with laboratory spectroscopy. In the initial stage of their identifications, a pure gas-phase model using cosmic ray ionisation and ion-molecule reactions seemed to be successful in explaining the existence of simple interstellar molecules. However, many heavier long carbon-chain molecules have been detected in dark clouds and circumstellar envelopes, and various saturated molecules and deuterated species have been found to exist in abnormally high abundances in star-forming regions. These observations suggest that the simple gas-phase reaction model is not sufficient, and contributions of dust-related reactions are essential in interstellar chemistry (Ohishi \& Kaifu 1998).

Discussions on interstellar chemistry have accelerated laboratory studies of heavier molecules with cm Fourier transform microwave spectroscopy (McCarthy, this volume). The heavy carbon-chain molecules connect gas-phase 
reactions to dust-related reactions. On the other hand, even now we are aware that there are many atomic and molecular species having transitions in the high frequency region and which may be useful in clarifying interstellar chemistry, especially in the boundary regions between molecular clouds and photodissociation regions. For example, new molecular ions, $\mathrm{CH}^{+}, \mathrm{OH}^{+}, \mathrm{SiH}^{+}, \mathrm{SH}^{+}, \mathrm{CH}_{2} \mathrm{D}^{+}$, $\mathrm{HCCD}^{+}, \mathrm{NH}_{3} \mathrm{D}^{+}, \mathrm{H}_{3} \mathrm{O}^{+}$, and simple hydrides, $\mathrm{CH}, \mathrm{SiH}, \mathrm{CaH}, \mathrm{NHD}, \mathrm{CH}_{2} \mathrm{D}$, $\mathrm{CH}_{3} \mathrm{D}, \mathrm{SiH}_{2}, \mathrm{SiH}_{2} \mathrm{D}$ have important transitions in the submillimeter-wave to far-infrared region.

When interstellar spectral lines are observed from cold dark clouds, one often needs a spectral resolution of $0.1 \mathrm{~km} \mathrm{~s}^{-1}$ which corresponds to only 10 $\mathrm{kHz}$ in the centimeter-wave range and to $1 \mathrm{MHz}$ in the terahertz range. Therefore, we must determine the line frequency with corresponding precision. Table 1 lists laboratory spectroscopic methods which can provide this requirement. The spectroscopic method of a combination of klystrons or Gunn diodes with multipliers is a powerful system with sufficiently high resolution (Saito 1991). It has been used at the Institute for Molecular Science for many years and has been very productive. The recently developed submillimeter-wave source, the Backward Wave Oscillator (BWO), is also a powerful tool up to $1.3 \mathrm{THz}$, as demonstrated by Russian scientists and G. Winnewisser's group in Köln (Winnewisser et al. 1994). The far-infrared laser magnetic resonance spectrometer and far-infrared sideband spectroscopy are unique methods for studying spectral lines in this region, but their accuracy ranges from several hundred $\mathrm{kHz}$ to a few $\mathrm{MHz}$ which is not sufficient for the spectral resolution required for observations of cold clouds. A tunable far-infrared spectrometer using Metal-Insulator-Metal (MIM) diodes, which has been developed by K. Evenson, is a versatile method covering a wide frequency range up to around $6 \mathrm{THz}$ with good precision (Nolt et al. 1987). However, the tunable power generated from an MIM diode is too small for high-sensitivity spectroscopy. Very recently, G.A. Blake and his collaborators have been developing a new photomixing frequency difference laser spectrometer, which is very compact and generates coherent radiation from several GHz to $1.6 \mathrm{THz}$ (Pearson et al. 1999).

To summarise, the development of laboratory spectroscopic methods is rapid and substantial, and interesting spectral lines such as those mentioned above, will be measured with sufficient precision in the very near future and will be used as new probes to investigate interstellar phenomena.

Table 1. High-resolution Spectroscopic Methods from $\mathrm{GHz}$ to THz.

\begin{tabular}{lccc}
\hline Method & $\begin{array}{c}\text { Frequency range } \\
(\mathrm{GHz})\end{array}$ & $\begin{array}{c}\text { Precision } \\
(\mathrm{MHz})\end{array}$ & Sensitivity \\
\hline Kly, Gunn + Mult. & $\sim 900$ & $<0.1$ & High \\
Phase-locked BWO & $\sim 1300$ & $<0.1$ & High \\
FIR-LMR & $\sim 6000$ & $1-3$ & Very high \\
Sideband FIR laser & $\sim 2500$ & $1-3$ & Medium \\
MIM diff. laser & $\sim 6000$ & $<0.1$ & Low \\
Photomix. diff. laser & $\sim 1600$ & $<0.1$ & Medium \\
\hline
\end{tabular}




\section{Molecular Spectroscopy in the VUV/UV/Visible (F. Rostas)}

With the advent of millimeter wave radioastronomy and, more recently, that of infrared and submillimeter space observatories, optical spectroscopy has ceased to be the dominant source of information concerning the physical parameters and the composition of astrophysical media. Nevertheless the need for spectroscopic data concerning molecules in the wavelength ranges extending from the EUV to near IR has not decreased by any means, in particular as they are valuable indicators of the photodissociation and photoionization dynamics. Reference to recent spectoscopic work of astrophysical interest can be found in the report of Working Group 5 of IAU Commission 14 (van Dishoeck \& Black 1999; see also $\langle$ http://www.obspm.fr/IAU14>).

\subsection{Species identification}

The main need is for precise line positions and spectrum analyses. New demands have appeared due to the increase in observational performances both in resolution and sensitivity. Spectra of radicals and ions are strongly needed. A recent examples is $\mathrm{C}_{7}^{-}$, a suggested candidate for some of the diffuse interstellar bands (Tulej et al. 1998).

\subsection{Radiation transfer}

Rotationally-resolved absorption spectra are needed in order to model the interaction of radiation with molecule-containing media. This is the case in particular when photochemistry is studied in planetary atmospheres, comets and the ISM. In this respect the spectra of such basic molecules as $\mathrm{N}_{2}, \mathrm{SO}_{2}, \mathrm{NO}$, or $\mathrm{O}_{2}$ are insufficiently known. In stellar atmospheres molecular opacities can be very important and participate in the energy balance. In these applications a huge number of lines has to be considered, especially for heavy molecules. The accent is more on line intensities than on precise positions and the line shapes due to predissociations and collisions play an important role. A recent study of this type concerns TiO.

\subsection{Spectral simulations}

These are needed not only, as above, for radiation transfer calculations but also for diagnostic purposes (particularly in interstellar and circumstellar media) where curves of growth have to be calculated taking into account instrument width effects. These applications are the most exacting as they require precise line positions, intensities and shapes.

\subsection{New or developing laboratory techniques}

In the laboratory, significant progress can be made by new developments in experimental techniques. These include advances in VUV Fourier Transform Spectrometers, to give improved precision, sensitivity and resolution (Yoshino et al. 1998); in Cavity Ringdown Spectroscopy (CRDS), which is very useful for weak absorption spectra and/or low sample density situations (Wheeler et al. 1998). Improvements in femtosecond laser techniques (Zewail 1996), will aid the study of molecular dynamics, transition states, and act as benchmark 
experiments to test calculations. The study of highly excited Rydberg states and photoionization processes will be improved by the use of ZEKE (Zero Kinetic Energy) ion spectroscopy (Merkt 1997).

\subsection{Some trends}

Astrophysical observations, particularly those involving large molecules and dust-related species, will lead to the increasing importance of ab-initio calculations and spectral simulation programs, particularly to aid molecular identification, to the study of photochemistry on grain surfaces and the related issue of photodesorption as a mechanism for returning surface species to the gas phase, and to the investigation of the spectroscopy and photodissociation of radicals and ions.

\subsection{Databases}

Once fundamental data has been obtained there is a pressing need to make them available to the astrophysical community. In particular one needs critically evaluated data, similar to the NIST tables for atoms and ions. There is also a pressing need for compilations of molecular constants; for example, an update of the Huber \& Herzberg (1979) tables would be very useful for spectroscopy. Tabulations of line positions and strengths are also required. The existing databases (Kurucz or Jørgensen) are oriented towards stellar atmospheres. For planetary atmospheres, there are no equivalent of HITRAN and GEISA databases for electronic transitions (e.g. Rothman et al. 1998; Jacquinet-Husson et al. 1998). Finally, one needs information on predissociation and collisional line shapes.

\subsection{Personnel matters}

It should be stressed that electronic molecular spectroscopy and photodynamics tend to be neglected as far as support and career opportunities are concerned. There is a real danger that the presently active generation of scientists might not be replaced in due time and that their knowledge could disappear rapidly.

\section{Modelling Astrochemistry (T. J. Millar)}

In recent years, there has been a noticeable increase in the number of research groups using astrochemical models to interpret observational data. To date, two models specifically developed for studying chemical evolution in interstellar clouds, those from UMIST 〈http://www.rate99.co.uk $>$ and Ohio State University, <http://www. physics .ohio-state .edu/ terzieva/NSM/Readme . html>, are widely used, and are publicly available. Both models can be applied to circumstellar envelopes, although specific codes for this application have been released by Mamon \& Glassgold <http://www.iap.fr/users/gam/csenv.html>. Furthermore, MHD shock models, developed by Flower, Pineau des Forêts \& Hartquist, are also available to the community $\langle\mathrm{http}: / / \mathrm{ccp} 7$.dur.ac.uk/>. All of these models are based on gas-phase chemistry and some can involve up to 10000 reactions among some 1000 species (e.g. Bettens \& Herbst 1997). It is worth recalling that the paper of Herbst \& Klemperer (1973), which is generally acknowledged as the seminal paper in the development of time-dependent 
chemical kinetics of interstellar clouds, included around 100 species coupled by some 500 reactions. These gas-phase models have had considerable success in accounting for the fractionation observed in deuterated molecules, the observations of ions, and the preponderance of carbon-chain molecules in interstellar and carbon-rich circumstellar envelopes.

The development of such large models is due entirely to increasing computer power. However, a significant fraction of reactions have never been studied at low temperatures, although for highly exothermic ion-neutral reactions, the rate coefficients can be estimated with some accuracy. Nevertheless, one important aim of Astrochemistry in the next few years will be to determine which of these 10000 reactions can be omitted without loss of accuracy. Of course, the results will differ depending on the nature of the region being investigated, and on the evolution of the system in time, so one needs some semi-automatic method of doing this. D. Howe at UMIST has gone some way towards this in recent years. In his method he compares a complete chemistry with models built up by including those reactions which dominate formation and destruction of individual species up to some prescribed level of accuracy. Although this can be time consuming in its initial stages, the CPU savings made if several hundred calculations need to be performed, as for example in depth-dependent models, or in models with two or three spatial dimensions, make such an approach very efficient.

In addition to excluding unimportant reactions, one also wants to identify critical reactions, that is those reactions for which small changes to their rate coefficients can alter particular abundances by a large amount. This would enable the important reactions to be identified for further theoretical and experimental study. Such a sensitivity analysis is used routinely in combustion chemistry and could be incorporated readily into astrochemical calculations since it requires information already available in the GEAR-type integrators normally used to solve the systems of rate equations.

In the last decade, it has been recognised that the gas-grain interaction can be important in dense gas, particularly through the depletion of atoms and molecules, and through the formation and evaporation of ice mantles in starforming regions. Details of the surface chemistry are still very uncertain. Typically, 100-200 such reactions may be included in a numerical model but there is a great paucity of laboratory data. The detection of large abundances of hydrogenated molecules in hot cores indicates that surface chemistry is very complex (Millar, Macdonald, \& Gibb 1997). Indeed there is still debate over the correct method to use for surface chemistry (see Shalabiea, Caselli, \& Herbst 1998). An additional uncertainty is that there is no agreed set of binding energies, which will of course differ depending on the matrix in which atoms and molecules are bound. Since desorption rates and surface mobilities depend exponentially on binding energies, small differences in them can produce large differences in the calculated abundances of both surface and gas-phase species.

Further information needed in relation to grain chemistry and desorption includes (i) determining activation energy barriers, particularly for reactions involving $\mathrm{H}, \mathrm{D}$ and $\mathrm{H}_{2}$, (ii) determining the effect of excess chemical energy in desorbing products and nearby species, (iii) investigating the efficiency of desorption following absorption of energetic photons and cosmic ray particles, (iv) investigating the formation of complex species from simple ice mixtures, and 
(v) determining the products of the interaction of molecular ions with negatively charged grains.

Finally, it is worth recalling that much interest is now being given to astrochemistry in regions far removed physically from the cold, dense clouds studied traditionally. In particular, chemistry in protoplanetary disks (Aikawa et al. 1996, 1997, 1999; Bauer et al. 1997; Finocchi, Gail, \& Duschl 1997; Willacy et al. 1998) involve densities up to $10^{16} \mathrm{~cm}^{-3}$ for which three-body reactions are important and for which temperatures of up to $1500 \mathrm{~K}$ mean that collisional dissociation is important. Another interesting application of astrochemistry in extreme situations is found in the study of dust formation, in particular the formation of small clusters, in the hydrogen-poor winds of Wolf-Rayet stars (Cherchneff et al. 2000). These objects have winds of several thousand $\mathrm{km} \mathrm{s}^{-1}$ and are observed to form dust from what appears to be completely ionized gas. The determination of rate coefficients at several thousand degrees, particularly for species containing carbon and silicon, is a formidable challenge for the future.

\section{Concluding Remarks}

Significant funds continue to be invested in hardware necessary for the development of molecular astrophysics which, as we have seen in this Symposium, has the ability to provide solutions to fundamental questions on all scales from galaxy formation, to star formation, to planet formation. However, to fully realize this potential, it is important that the requisite fundamental data are available. The provision of such data and the development of theoretical models is much less expensive than hardware, but it is clearly a common experience that, when finances are scarce, funding agencies are likely to reduce support for these areas. It is important for all scientists involved in Astrochemistry and molecular astrophysics to support adequate levels of funding for data provision and modelling.

\section{References}

Aikawa, Y., Miyama, S. M., Nakano, T., \& Umebayashi, T. 1996, ApJ, 467, 684 Aikawa, Y., Umebayashi, T., Nakano, T., \& Miyama, S.M. 1997, ApJ, 486, L51 1999, ApJ, 519, 705

Balakrishnan, N., Forrey, R.C., \& Dalgarno, A. 1999, ApJ, 514, 520

Bauer, I., Finocchi, F., Duschl, W.J., Gail, H.-P., \& Schlöder, J.P. 1997, A\&A, 317,273

Bettens, R.P.A. \& Herbst, E. 1997, ApJ, 478, 585

Cherchneff, I., Le Teuff, Y.H., Williams, P.M., \& Tielens, A.G.G.M. 2000, A\&A, in press

Dartois, E., et al. 1998, A\&A, 331, 651

de Graauw, T., et al. 1996, A\&A, 315, L345

Dunning, F.B. 1995, J. Phys. B: At. Mol. Opt. Phys., 28, 1654

Finocchi, F., Gail, H.-P., \& Duschl, W.J. 1997, A\&A, 325, 1264

Flower, D.R. 1990, Molecular Collisions in the Interstellar Medium (Cambridge: Cambridge University Press) 1998, MNRAS, 297, 334 
Flower D.R., Offer A., \& Schilke, P. 1990, MNRAS, 244, 4

Galli, D. \& Palla, F. 1998, ApJ, 521, 630

Guillois, O., Ledoux, G., \& Reynaud, C. 1999, ApJ, 521, L133

Guilloteau, S., Omont, A., Cox, P., McMahon, R.G., \& Petitjean, P. 1999, A\&A, 349,363

Guilloteau, S., Omont, A., McMahon, R.G., Cox, P., \& Petitjean, P. 1997, A\&A, $328, \mathrm{~L} 1$

Herbst, E. \& Klemperer, W. 1973, ApJ, 173, 505

Huber, K.P. \& Herzberg, G. 1979, Constants of Diatomic Molecules (New York: Van Nostrand Reinhold)

Hutchins, J.B. 1976, ApJ, 205, 103

Jacquinet-Husson, N., et al. 1998, JQRST, 59, 511

Johnston, K.J., Gaume, R., Stolovy, S., Wilson, T.L., Walmsley, C.M., \& Menten, K.M. 1992, ApJ, 385, 232

Lees, R.M. \& Hague, S.S. 1974, Canad. J. Phys., 52, 2250

Merkt, F. 1997, Ann. Rev. Phys. Chem., 48, 66

Millar, T.J., Macdonald, G.H., \& Gibb, A.G. 1997, A\&A, 325, 1163

Mitchell, J.B.A. \& Rebrion-Rowe, C. 1997, Int. Rev. Phys. Chem., 16, 201

Nolt, I.G., Radostitz, J.V., DiLonardo, G., et al. 1987, J. Mol. Spectrosc., 124, 274

Ohishi, M. \& Kaifu, N. 1998, Faraday Dis., 109, 205

Pearson, J.C., Pickett, H.M., Matsuura, S., \& Blake, G.A. 1999, The 54th Ohio State Univ. Int. Sym. Mol. Spectrosc. WF01

Rothman, L.S., et al. 1998, JQSRT, 60, 665

Roueff, E. \& Flower, D.R. 1999, MNRAS, 305, 353

Saito, S. 1991, Astr. Soc. Pacific Conf. Ser., 16, 349

Shalabiea, O.M., Caselli, P., \& Herbst, E. 1998, ApJ, 502, 652

Shapiro, P.R. \& Kang, H. 1987, ApJ, 318, 32

Smith, I.W.M., Rowe, B.R., \& Sims, I.R. 1996, in Gas-Phase Chemical Reaction Systems: Experiments and Models 100 Years after Max Bodenstein, eds. J. Wolfrum, H.R. Volpp, R. Dannacher \& J. Warnatz (Berlin: Springer), 190

Smith, M.A. 1994, in Unimolecular and Bimolecular Ion-Molecule Reaction Dynamics, eds. C.Y. Ng, T. Baer, \& I. Powis (Chichester: J. Wiley Publ.), 251

Troe, J. 1996, J. Chem. Phys., 105, 6249

Tulej, M., Kirkwood, D.A., Pachkov, M., \& Maier, J.P. 1998, ApJ, 506, L69

van Dishoeck, E. \& Black, J.H. 1999, in Reports in Astronomy vol. XXIV, ed. J. Andersen (ASPS: San Francisco)

Waters, L.B.F.M., et al. 1996, A\&A, 315, L361

Wheeler, M.D., Newman, S.M., Orr-Ewing, A.J., \& Ashfold, M.N.R., J. 1998, Chem. Soc. Faraday Trans., 94, 337

Willacy, K., Klahr, H., Millar, T.J., \& Henning, Th. 1998, A\&A, 338, 995

Winnewisser, G., Krupnov, A.F., Tretyakov, M.Yu., Liedtke, M., Leven, F., Saleck, A.H., Schieder, R., Shkaev, A.P., \& Volokhov, S.A. 1994, J. Mol. Spectrosc., 165, 294

Wyrowski, F., Schilke, P., \& Walmsley, C.M. 1999, A\&A, 341, 882

Yoshino, K., Esmond, J.R., Parkinson, W.H., et al. 1998, J. Chem. Phys., 109, 1751

Zewail, A.H. 1996, J. Phys. Chem., 100, 12701 